\title{
An Uncommon Presentation of Amyloidosis
}

\author{
Brandon Kujawski, MS III, Drew Johnson, MS III, Kushan Radadia, MS IV, Eric Feduska, MD, \\ Robert Ford, MD, Guldeep Uppal, MD, Mariam Kabir, MD,PhD, John Stewart, MD
}

\section{INTRODUCTION}

AL amyloidosis is a rare disease, with only 1200-3200 new cases in the US per year. ${ }^{1}$ Two-thirds of patients are male; presentation typically occurs after age fifty. ${ }^{1.2,3}$ Amyloid can involve the kidneys (74\%), heart (60-90\%), liver $(27 \%)$, peripheral nervous system (22\%), and carpal tunnel (20\%). ${ }^{3}$ We describe an atypical presentation of $\mathrm{AL}$ amyloidosis and highlight the importance of recognizing this disease in patients with systemic signs.

\section{CASE PRESENTATION}

A 60 year old Caucasian male was transferred from an outside hospital with four months of progressive lower extremity burning pain and weakness, unintentional 34-pound weight loss, fatigue, subjective fevers, night sweats and intermittent blood in his stool. Review of systems was otherwise negative. Medical, surgical, and family history were unremarkable; he took no medications. Social history was notable for heavy alcohol use until four months prior to admission.

At the outside hospital, the patient had undergone a thorough workup for his Gl bleeding, which included an MRI of his abdomen, esophagogastroduodenoscopy, and colonoscopy. Hepatomegaly was noted on the abdominal MRI, and several internal hemorrhoids were seen on the colonoscopy.

Vital signs were within normal limits. Physical exam was notable for cachexia, hepatomegaly, and 3-out-of-5 strength in the upper and lower extremities. The remainder of the exam was normal.

Laboratory evaluation showed a hemoglobin of $8.0 \mathrm{~g} /$ $\mathrm{dL}$ (normal range $=14-17 \mathrm{~g} / \mathrm{dL}$ ) with a normal mean corpuscular volume, ferritin of $997 \mathrm{ng} / \mathrm{mL}$ (normal range $=50-150 \mathrm{ng} / \mathrm{mL}$ ), iron of $29 \mathrm{mcg} / \mathrm{dL}$ (normal range $=55-160 \mathrm{mcg} / \mathrm{dL}$ ), and total iron binding capacity of $176 \mathrm{mcg} / \mathrm{dL}$ (normal range $=250-400 \mathrm{mcg} / \mathrm{dL}$ ),

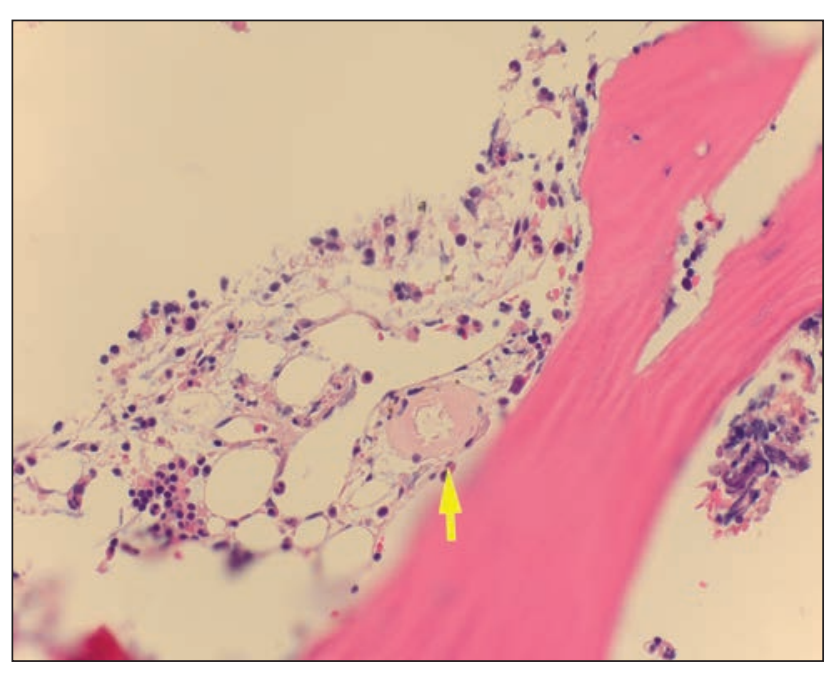

Figure 1. Bone marrow biopsy showing a vessel with deposition of pink amorphous material in the wall (Hematoxoylin \& Eosin, original magnification $400 \mathrm{X}$ ). There was no evidence of a neurogenic process or malignancy.

indicating anemia of chronic disease. White blood cell count was $9.0 \times 10^{\%} / \mathrm{L}$ (normal range $=4-11 \times 10^{\%} / \mathrm{L}$ ) with a normal differential. Platelet count was $1167 \times 10 \% / \mathrm{L}$ (normal range $=140-400 \times 10 \% / L$ ). Blood smear showed Howell-Jolly bodies and thrombocytosis with reactive platelets. His electrolytes and creatinine clearance were normal. Prothrombin time (PT) was elevated at $36.9 \mathrm{sec}$ (normal range $=9.4-12.4 \mathrm{sec}$ ), and this did not correct with Vitamin K (oral or subcutaneous). His partial thromboplastin time (PTT) was high at $51 \mathrm{sec}$ (normal range $=28-38 \mathrm{sec}$ ). Both $\mathrm{PT}$ and $\mathrm{PTT}$ corrected with mixing studies indicating a factor deficiency.

Hepatic panel revealed an alkaline phosphatase of 563 IU/L (normal range $=25-150 \mathrm{IU} / \mathrm{L}$ ). He was negative for HIV, Hepatitis B and C, and syphilis. Serum protein electrophoresis (SPEP) showed hypogammaglobulinemia with an abundance of lambda light chains; urine protein electrophoresis (UPEP) showed a free lambda level of $12.26 \mathrm{mg} / \mathrm{dL}$ (normal range $=0.02-0.67 \mathrm{mg} /$ 


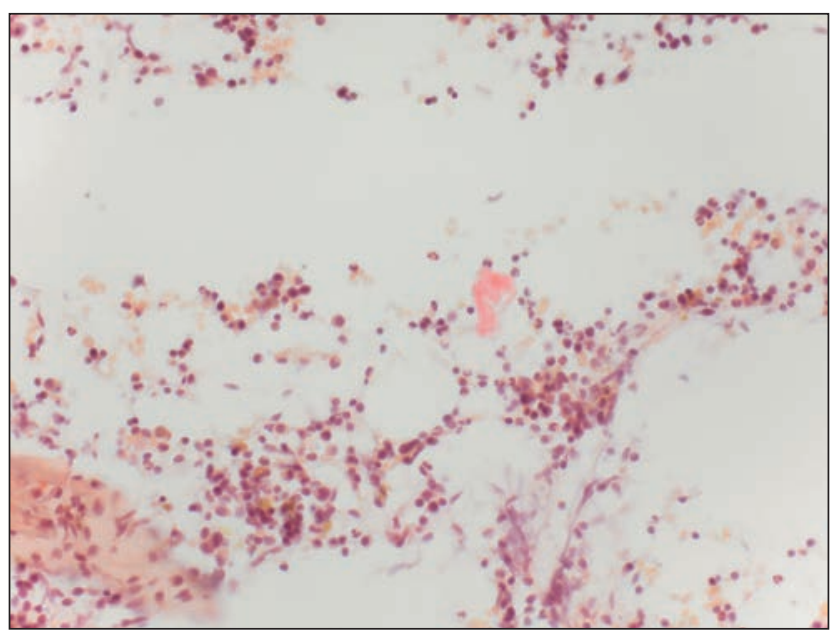

Figure 2 \& 3 . Bone marrow biopsy showing Congo Red staining polarized light (Figure 3) (Congo Red stain, original magnification 200X).

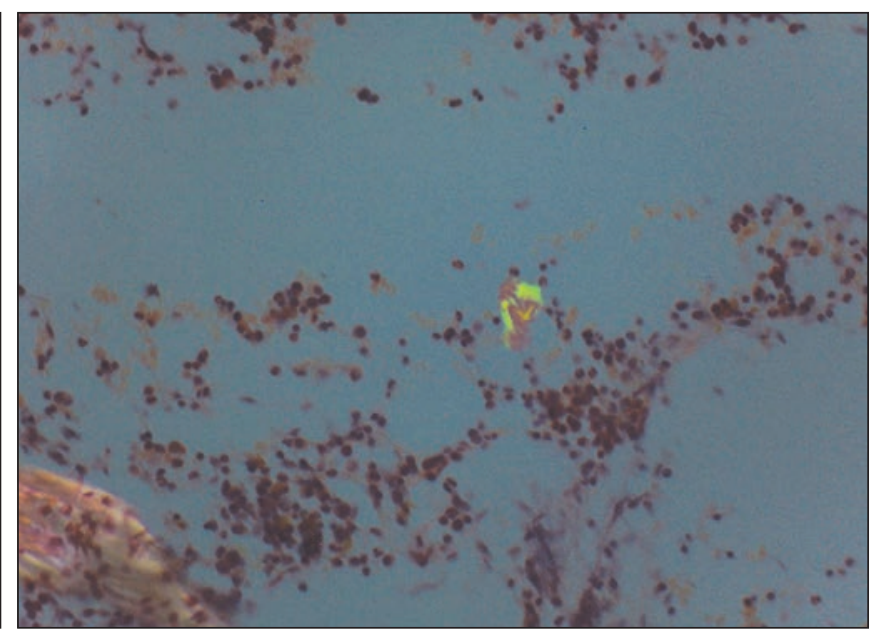

Figure 2) and apple green birefringence in the vessel wall under $\mathrm{dL}$ ) indicating a likely plasma cell dyscrasia with lambda overproduction. Pro-B-type natriuretic peptide (pro-BNP) was $3936 \mathrm{pg} / \mathrm{mL}$ (normal range = <100 pg/mL).

CT scans of the thorax, abdomen, and pelvis were unremarkable except for an enlarged liver. His bone scan and skeletal survey were negative for lytic lesions. Electrocardiogram showed normal sinus rhythm with intraventricular conduction delay, first-degree AV block, and low voltage throughout. A trans-thoracic echocardiogram showed an ejection fraction of $60 \%$ with impaired relaxation of the left ventricle. There was no speckled pattern in the myocytes and no restrictive cardiomyopathy.

\section{DIFFERENTIAL DIAGNOSIS}

Our differential diagnosis included myelofibrosis, essential thrombocytosis, and reactive thrombocytosis from a systemic disease such as inflammatory bowel disease or amyloidosis. Although multiple myeloma was also a concern, the absence of lytic lesions, hypercalcemia and renal failure essentially ruled it out. ${ }^{4,5}$ Given the patient's presentation and laboratory abnormalities, Hematology was consulted.

The patient was negative for the JAK2V617F mutation, making myelofibrosis or essential thrombocytosis unlikely. ${ }^{6}$ Given the presence of a monoclonal protein on SPEP and UPEP, factor $X$ deficiency (5\%), and the presence of immunoglobulin light chains in the serum and urine, a systemic fibril deposition disease such as amyloidosis was most likely. Bone marrow biopsy was performed, which showed an infiltrate of monoclonal lambda positive plasma cells (5\%) on immunohistochemical staining, with deposition of amyloid fibers in blood vessels (Figure 1). Congo red stain showed apple-green birefringence under polarized light (Figures 2-3). This led to the diagnosis of light chain amyloidosis.

\section{OUTCOME AND FOLLOW-UP}

The patient was discharged to a subacute rehabilitation facility with plan to follow-up with a hematologist/ oncologist within one week of discharge. No treatment plans were made during his hospital stay due to his level of deconditioning. Since his gastrointestinal bleeding was minimal, intermittent, and he never required blood transfusion, his factor deficiency did not require correction.

\section{DISCUSSION}

Amyloidosis is caused by the abnormal deposition of amyloid protein in a single or multiple organs. Sixty different types of amyloid protein have been identified so far, and thirty-six have been connected with deposition disease. ${ }^{7.8}$ For example, AA amyloidosis is caused by the deposition of an acute phase reactant protein in various organs. This acute phase reactant is usually generated in the setting of inflammatory disease (inflammatory bowel disease, rheumatoid arthritis, etc) or chronic infection (tuberculosis, chronic osteomyelitis, etc). ${ }^{9} \mathrm{AL}$ 
amyloidosis, on the other hand, is due to deposition of protein derived from immunoglobulin light chain fragments. It is a plasma cell dyscrasia that can occur alone or with multiple myeloma, Waldenstrom's macroglobulinemia, or non-Hodgkin's lymphoma. ${ }^{2}$

The diagnostic criteria for AL amyloidosis include the presence of an amyloid-related systemic syndrome, positive amyloid staining by Congo red in any tissue, evidence that the amyloid is light-chain related, and evidence of a monoclonal plasma cell proliferative disorder. ${ }^{2}$ Although the most definitive proof of light chain amyloid is by mass spectrometry of a biopsy sample, immunohistochemical staining of the sample can also be used for diagnosis. ${ }^{10}$ Our patient met all of the above criteria, as follows. He had evidence of liver involvement, cardiac involvement (low voltages, first degree AV block), and likely peripheral neuropathy. There was evidence of amyloid by Congo red staining. Immunohistochemical staining of his bone marrow showed plasma cells with lambda light chain predominance. In addition, he had excessive lambda light chains in his serum and urine, indicating monoclonal plasma cell proliferation. We did not send a sample for mass spectrometry.

AL amyloidosis with liver involvement has several interesting features. A review of patients with $\mathrm{AL}$ amyloidosis analyzed a total of 98 patients with hepatic involvement. ${ }^{11}$ Among these, $71 \%$ had weight loss and hepatomegaly; our patient had both of these. Laboratory studies showed alkaline phosphatase elevations in $86 \%$ of patients; AST/ALT were only elevated in $37 \%$ of patients. Our patient only had an alkaline phosphatase elevation. Amyloidosis also led to functional hyposplenism with reactive thrombocytosis in $~ 28 \%$ of patients. A classic hematologic finding in these patients on peripheral blood smear was the presence of Howell-Jolly bodies, as were seen on our patient's blood smear.

A unique finding in our patient was the prolonged PT with associated gastrointestinal bleeding, which was found to be secondary to factor $X$ deficiency. A study looking at acquired factor $X$ deficiency in patients with AL amyloidosis, found that 32/368 (8.7\%) had factor $X$ deficiencies. ${ }^{12}$ Another study of 337 patients done by Mumford et a $1^{10}$ found that $172 / 337$ had prolonged PT, PTT, or thrombin time. Cutaneous bleeding occurred in 60/337 (18\%), and gastrointestinal bleeding occurred in $16 / 337(5 \%) .10$

Treatment choices for AL amyloidosis include melphalan or cyclophosphamide in conjunction with dexamethasone. ${ }^{13}$ Stem cell transplant (SCT) is offered to patients who fit the criteria, which include age $<70$, troponin $<0.06$, pro-BNP $<5000$, creatinine clearance >30, NYHA Class I or II heart failure, no more than two organs involved, no large pleural effusion, and no hypoxia. ${ }^{13}$

The prognosis of patients with AL amyloidosis is poor. Without treatment, the median survival is 13 months. ${ }^{13.14}$ An Italian study of $705 \mathrm{AL}$ amyloidosis patients who underwent treatment showed a median survival of 46 months. A UK study of 600 patients with treatment showed a median survival of 2-3 years. ${ }^{15,16}$

\section{KEY POINTS}

We present a patient with amyloid deposition disease, discuss criteria for the diagnosis of AL amyloidosis, and demonstrate how our patient met these criteria. In addition, we point out that although AL amyloidosis most commonly involves the kidney and the heart, there is a less common pattern that affects the liver and causes hepatomegaly, coagulopathy and bleeding. It is important to recognize this pattern and provide timely treatment. In addition, we stress that although there are treatment options available for AL amyloidosis, the prognosis, even with treatment, is poor.

\section{REFERENCES}

1. Primary Amyloidosis. (Accessed January 17, 2014, at http://www amyloidosis.org/TreatmentInformation/primaryAL.html)

2. AL Amyloidosis. (Accessed January 17, 2014 at http://www.unckidneycenter.org/kidneyhealthlibrary/amyloidosis.html)

3. Dubrey SW, Hawkins PN, Falk RH. Amyloid diseases of the heart: assessment, diagnosis, and referral. Heart. 2011; 97(1): 75-84.

4. International Myeloma Working Group. Criteria for the classification of monoclonal gammopathies, multiple myeloma and related disorders: a report of the International Myeloma Working Group. Br. J. Haematol. 2003; 121 (5): 749-57.

5. Kyle RA, Rajkumar SV. Criteria for diagnosis, staging, risk stratification and response assessment of multiple myeloma. Leukemia. 2009; $23: 3$.

6. Mascarenhas JO, Orazi A, Bhalla KN, Champlin RE, Harrison C, Hoffman R. Advances in myelofibrosis: A clinical case approach. Haemetologica. 2013; 98: 1499-1509. 
7. Mok KH, Pettersson J, Orrenius S, Svanborg C. HAMLET, protein folding, and tumor cell death. Biochem. Biophys. Res. Commun. 2007; 354 (1): 1-7.

8. Pettersson-Kastberg J, Aits S, Gustafsson L, et al. Can misfolded proteins be beneficial? The HAMLET case. Ann. Med. 2008; 41 (3): 1-15

9. Lachmann HJ, Goodman HJ, Gilbertson JA, et al. Natural history and outcome in systemic AA amyloidosis. New England Journal of Medicine. 2007; 356 (23): 2361-71

10. Schönland SO, HegenbartU, BochtlerT, etal. Immunohistochemistry in the classification of systemic forms of amyloidosis: a systematic investigation of 117 patients. Blood. 2012; 119(2): 488-93.

11. Park MA, Mueller PS, Kyle RA, Larsen DR, Plevak MF, Gertz MA Primary (AL) hepatic amyloidosis: Clinical features and natural history in 98 patients. Medicine (Baltimore). 2003; 82: 291-298.

12. Choufani EB, Sanchorawala V, Ernst T, Quillen K, Skinner M Wright DG, Seldin DC. Acquired factor $X$ deficiency in patients with amyloid light-chain amyloidosis: incidence, bleeding manifestations, and response to high-dose chemotherapy. Blood. 2001; 97: 1885-1887
13. Mumford A, O'Donnell J, Gillmore JD, Manning RA, Hawkins PN, Laffan M. Bleeding symptoms and coagulation abnormalities in 337 patients with AL-amyloidosis. British Journal of Haematology. 2000; 110(2): 454-60

14. Wechalekar AD, Hawkins PN, Gillmore JD. Perspectives in treatment of $\mathrm{AL}$ amyloidosis. British Journal of Haematology. 2008; 140 (4): 365-377

15. Kyle RA, Gertz MA, Greipp PR, et al. Long-term survival (10 years or more) in 30 patients with primary amyloidosis. Blood. 1999; 93: 1062-1066

16. Merlini G, Stone MJ. Dangerous small B-cell clones. Blood. 2006; 108: 2520-2530

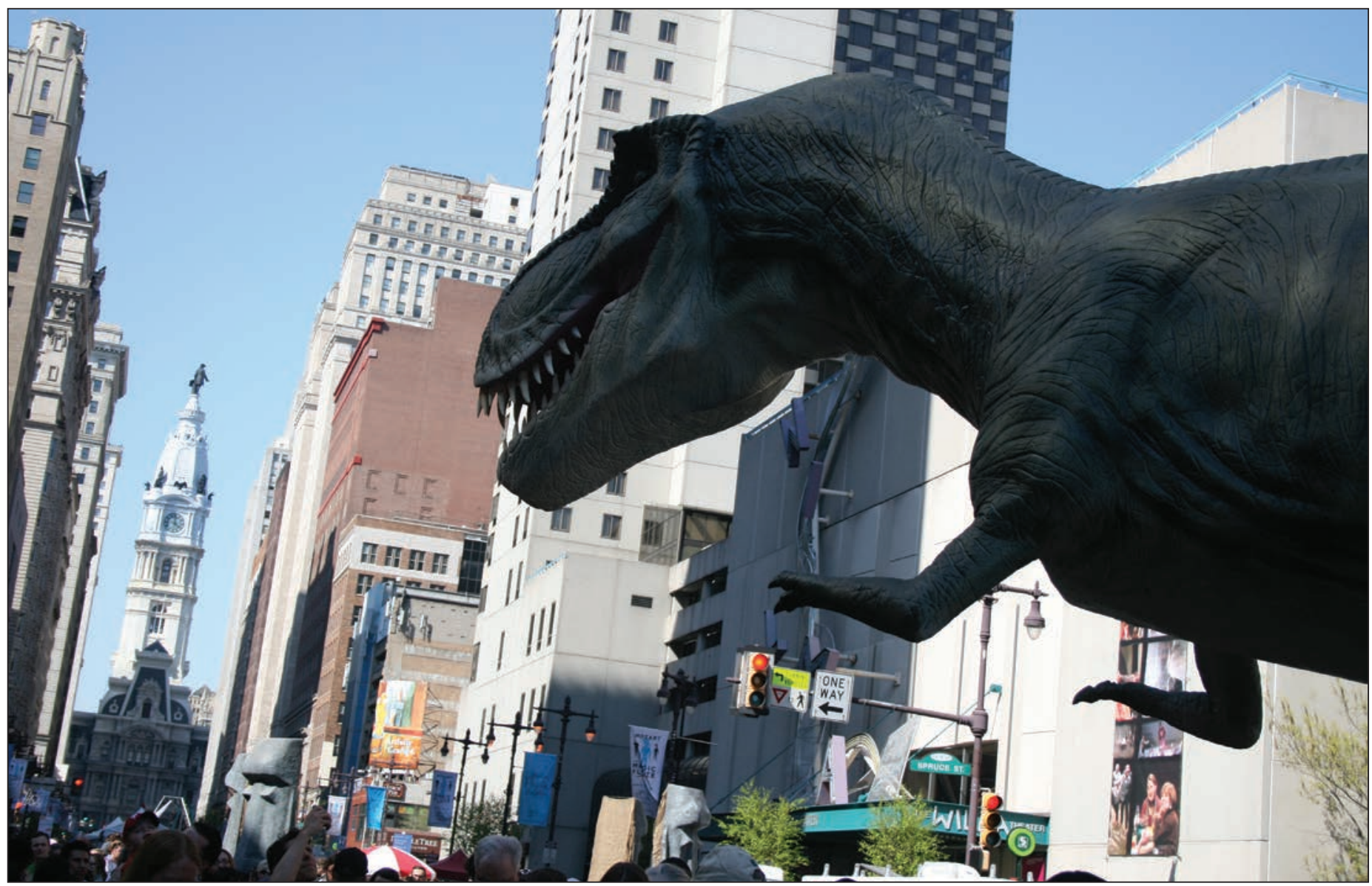

